\title{
Du court au long terme : un scénario «norvégien » pour pérenniser la rente nickel en Nouvelle- Calédonie?
}

Short to long term, a Norwegian-like scenario to perpetuate the nickel rent in New Caledonia

\section{Gaël Lagadec et Olivier Sudrie}

\section{OpenEdition \\ Journals}

Édition électronique

URL : http://journals.openedition.org/developpementdurable/9884

DOI : 10.4000/developpementdurable.9884

ISSN : 1772-9971

Éditeur

Association DD\&T

Référence électronique

Gaël Lagadec et Olivier Sudrie, «Du court au long terme : un scénario « norvégien » pour pérenniser la rente nickel en Nouvelle-Calédonie? », Développement durable et territoires [En ligne], Vol. 4, $n^{\circ}$

3 | Octobre 2013, mis en ligne le 30 octobre 2013, consulté le 30 avril 2019. URL : http://

journals.openedition.org/developpementdurable/9884; DOI : 10.4000/developpementdurable.9884

Ce document a été généré automatiquement le 30 avril 2019

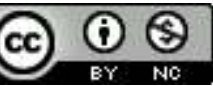

Développement Durable et Territoires est mis à disposition selon les termes de la licence Creative Commons Attribution - Pas d'Utilisation Commerciale 4.0 International. 


\title{
Du court au long terme : un scénario « norvégien » pour pérenniser la rente nickel en Nouvelle- Calédonie?
}

\author{
Short to long term, a Norwegian-like scenario to perpetuate the nickel rent in \\ New Caledonia
}

\section{Gaël Lagadec et Olivier Sudrie}

1 L'autonomie fiscale de la Nouvelle-Calédonie, préexistante depuis 1900, a été consacrée par l'article 9 de la loi no 88-1028 du 9 novembre 1988 portant dispositions statutaires et préparatoires à l'autodétermination de la Nouvelle-Calédonie, selon lequel «le Territoire est compétent dans les matières suivantes: $1^{\circ}$ les impôts, droits et taxes perçus dans le territoire (... )». Elle a été réaffirmée par l'article 22 de la loi organique n 99-209 du 19 mars 1999 relative à la Nouvelle-Calédonie selon lequel «la Nouvelle-Calédonie est compétente dans les matières suivantes: $1^{\circ}$ Impôts, droits et taxes perçus au bénéfice de la Nouvelle-Calédonie; création et affectation d'impôts et taxes au profit de fonds destinés à des collectivités territoriales, d'établissements publics ou d'organismes chargés d'une mission de service public; création d'impôts et taxes provinciaux ou communaux (...) ». Le $11^{\circ}$ de ce même article 22 prévoit également que la Nouvelle-Calédonie est compétente en matière de réglementation minière relative au nickel, au chrome et au cobalt.

Grâce à l'autonomie fiscale dont elle disposait, la Nouvelle-Calédonie a déjà pu dans le passé appliquer une fiscalité minière différente de celle de la Métropole (ainsi, il y a longtemps eu en Nouvelle-Calédonie une taxe à l'exportation qui n'existait pas en Métropole). Le nouveau code minier calédonien prévoit, depuis 2009, des dispositions originales, notamment sur la capacité du Pays à valoriser sa ressource.

Dans ce travail, la valorisation de la ressource est explicitement vue dans une perspective de développement durable : alors que l'activité minière détruit le capital naturel de la 
Nouvelle-Calédonie, comment assurer une préservation des revenus présents au profit de générations futures?

4 La première section insiste sur la volatilité de la fiscalité minière et sur ses conséquences négatives pour les collectivités (1.1.), notamment du fait des cycles politiques, dont il est montré à partir de la théorie des choix publics comment ils obèrent les capacités de développement de long terme (1.2.).

5 La seconde section étudie les possibilités de retraitement de la rente nickel en distinguant ce qui se fait actuellement en Nouvelle-Calédonie ainsi que les projets récurrents (2.1.) et, enfin, à partir d'une analyse des besoins futurs de la Nouvelle-Calédonie, en présentant les modalités de financiarisation de la rente sur le modèle pratiqué par la Norvège pour sa rente pétrolière (2.2.).

\section{La volatilité de la fiscalité minière}

\subsection{Constat}

\subsubsection{Les grands principes du budget calédonien}

6 La Nouvelle-Calédonie, en tant que collectivité territoriale, ne conserve qu'environ le quart des impôts et taxes qu'elle lève et encaisse. En d'autres termes, pour $1 €$ de financement supplémentaire requis par cette collectivité, l'application directe des textes amène souvent à augmenter de $4 €$ l'impôt versé par le contribuable ${ }^{1}, 3 €$ devant être redistribués à des collectivités tierces (provinces, communes), aux établissements publics ou à certains services publics. Cela signifie aussi qu'une baisse (ou une hausse) des recettes fiscales propres de la Nouvelle-Calédonie entraîne un effet en chaîne sur les recettes des autres collectivités. (Voir en annexe $\mathrm{n}^{\circ} 2$ le budget de répartition 2012 de la Nouvelle-Calédonie).

\subsubsection{Le rendement de l'IS 35}

7 La fiscalité minière repose quasi-exclusivement ${ }^{2}$ sur les profits des entreprises du secteur, à travers un impôt sur les bénéfices au taux de $35 \%$ (d'où son nom d'« IS 35 », par opposition à l'«IS 30 », qui s'applique à toutes les autres entreprises). Il n'existe en particulier aucune fiscalité sur l'extraction, ce qui, en pratique, fait du minerai une ressource gratuite pour les firmes qui l'exploitent. La fiscalité reposant sur l'IS, la recette est nulle quand les cours sont bas et que les entreprises ne font pas de bénéfice. Au contraire, lorsque les cours sont élevés, les profits des entreprises minières peuvent être colossaux, et la recette fiscale peut alors être très élevée.

8 Ainsi, à titre d'illustration, le rendement de l'impôt sur les sociétés minières et activités métallurgiques a été multiplié par 47 entre 2002 et 2007, passant de 0,5 à 23,5 milliards de francs $\mathrm{CFP}^{3}$ (CTC, 2008, p. 9). Le graphique $\mathrm{n}^{\circ} 1$ montre la volatilité du rendement de la fiscalité nickel. 
Graphique $n^{\circ} 1$. Évolution des recettes de la fiscalité avec et sans IS 35

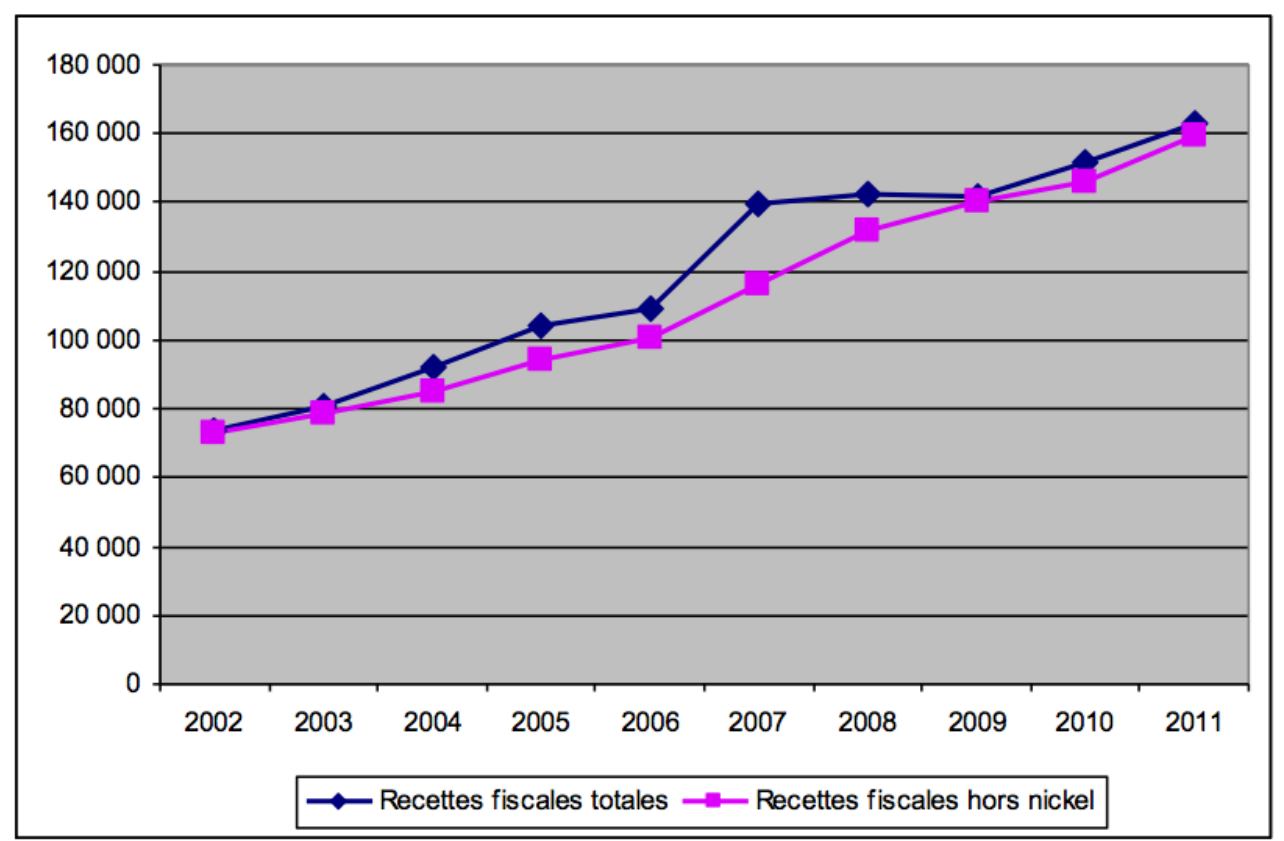

Source : Direction du budget et des affaires financières de la NC.

9 On constate que si en 2009 et 2010, les courbes des recettes fiscales avec et sans nickel se rejoignent; les recettes du nickel ont au contraire été importantes après 2004 pour culminer en 2007, avant de disparaître en 2009 (ce qui témoigne d'épisodes récents de volatilité extrême).

Le nickel apparaît ainsi comme une richesse relative pour la Nouvelle-Calédonie : son rendement fiscal est relativement faible et surtout très fluctuant et sa part dans le PIB et dans l'emploi total apparaît faible. Ce n'est qu'au niveau des exportations que le nickel est prépondérant, mais cet indicateur devrait être minoré des importations d'intrants nécessaires à l'activité et apparaît en outre avoir surtout une contrepartie en termes de profits, dont la Nouvelle-Calédonie bénéficie peu (Lagadec et Ris, 2011)4. Au total, le nickel génère un effet d'entraînement uniquement indirect sur le reste de l'économie : «L'impact mécanique de la hausse des cours du nickel sur le reste de l'économie est nul. Toutefois, son effet psychologique n'est pas négligeable; l'histoire récente montre que les variations des cours du nickel ne sont pas sans influence sur les anticipations des agents ainsi que sur le cycle des affaires " (GNC, 2010).

\subsection{Effet de cliquet des recettes fiscales sur la dépense publique}

\subsubsection{Effet de cliquet et cycles politiques}

11 L'assiette fiscale fluctue en fonction des cours mondiaux du nickel (cf. graphique $\mathrm{n}^{\circ} 1$ ). Cette volatilité pose un problème général pour les finances publiques puisque les dépenses augmentent régulièrement et que les dépenses de fonctionnement vérifient des effets de cliquet importants. Pour cette raison, la réforme fiscale en cours visera notamment à stabiliser l'assiette fiscale (c'est en tout cas l'objectif qui lui était régulièrement fixé dans le débat public local). Toutefois, la réforme risque d'être 
contrainte par le pacte de stabilité fiscale de la Nouvelle-Calédonie, qui tend à empêcher ${ }^{5}$ de modifier la fiscalité directe sur les sociétés minières. Toutefois, cet engagement de stabilité ne porte que sur les taxes, pas sur les redevances d'occupation du domaine public ou privé de la Nouvelle-Calédonie ou des collectivités, ni pour les redevances qui constituent la contrepartie de services rendus. C'est ainsi que la province Sud a, en 2008, fixé à un niveau élevé la redevance d'occupation du domaine maritime, pour l'implantation de l'émissaire de rejet des effluents de l'usine de Vale. Certains partis politiques ont également proposé la création d'une "redevance d'extraction minière " qui pourrait également participer à l'effort de solidarité intergénérationnelle. Cette proposition a été récemment reprise par Anne Duthilleul, missionnée par l'État et qui a livré en février 2012 son projet de rapport final sur l'avenir du nickel en Calédonie.

12 La volatilité de l'assiette se couple à un objectif de rendement. Ainsi, du fait de son autonomie institutionnelle croissante, la Nouvelle-Calédonie risque d'être confrontée à moyen et long terme à de nouveaux besoins. Les transferts de compétences en cours (enseignement secondaire, droit commercial, droit civil, sécurité civile), de même que les compétences déjà transférées (droit des assurances, règlementation économique, enseignement primaire et secondaire, droit du travail, etc.) sont en effet en principe compensés par l'État, mais d'une part cette compensation est dans certains secteurs notoirement insuffisante (sécurité civile) et d'autre part on peut craindre que les transferts de l'État diminuent à l'avenir, du fait de l'ampleur de sa dette. Il apparaît donc nécessaire d'augmenter, de façon pérenne, le rendement fiscal.

Le problème du cliquet peut se résumer à la question suivante: «que faire des bonnes surprises? ». Cette question se pose lorsque la conjoncture économique place les recettes fiscales au-delà des besoins immédiats.

Cette question est également indissociable des cycles politiques calédoniens, qui rythment, comme c'est aussi le cas ailleurs, la vie économique.

De façon générale dans la littérature sur le soutien politique, le parti au pouvoir utilise son monopole de l'offre des biens publics pour atteindre ses objectifs. Dans le cas calédonien, cette situation de monopole n'est limitée que par l'existence de partis d'opposition dans les provinces, et par le système collégial et de représentation proportionnel au gouvernement.

16 La littérature de l'école des choix publics propose deux modèles concurrents basés sur l'existence de cycles politico-économiques : le cycle opportuniste (qu'on étudie infra) et le cycle partisan ${ }^{6}$. Au sein de chaque modèle existe un postulat concernant d'une part le comportement des électeurs et d'autre part celui des élus.

17 Dans le cycle opportuniste, la théorie des choix publics fait l'hypothèse que le comportement des élus est une réponse au comportement des électeurs. Or, le résultat des votes dépend essentiellement de la situation économique d'un pays au moment des élections, les électeurs ont tendance à sanctionner (reconduire) le candidat sortant lorsque les performances de l'économie sont jugées défavorables (satisfaisantes).

18 Les élus vont donc tenter de manipuler les instruments de la politique économique de façon à accroître la probabilité de leur victoire aux prochaines élections. Le gouvernement est ainsi traditionnellement défini, dans l'approche des choix publics, par son comportement « chercheur de réélection » (Austen-Smith et Wright 1992, p. 230, par exemple). 
19 Pourtant l'homme politique peut avoir des objectifs autres que conserver et exercer le pouvoir. Le premier d'entre eux est la formation et la mise en œuvre de la politique gouvernementale. Breton (1981) considérait ainsi que l'objectif premier de l'homme politique est non pas la réélection mais la maximisation du pouvoir (Breton 1981, p. 360).

Ce pouvoir $(P)$ est défini comme la différence entre la probabilité de réélection $(\pi)$ et la probabilité seuil $\left(\pi^{*}\right)$, c'est-à-dire la probabilité suffisante pour que le gouvernement gagne l'élection à venir (plus précisément, ce doit être la probabilité à partir de laquelle le gouvernement n'envisage même pas de perdre l'élection).

21 Le gouvernement doit donc maximiser $P=\pi-\pi^{*}$. $P$ peut être augmenté (dans l'analyse de Breton) par la mise en œuvre de politiques appréciées par des citoyens, et qui signalent cette préférence. $P$ peut être diminué de deux manières : premièrement par des erreurs de politique gouvernementale, qui diminuent $\pi$; et deuxièmement en mettant sciemment en œuvre des politiques que les hommes politiques au pouvoir apprécient pour euxmêmes, mais que les citoyens réprouvent (Breton 1981, p.361) et qui peuvent être motivées par la recherche d'une «rente égotiste» de la part des hommes politiques (Farvaque et Paty, 2009, p. 40).

L'auteur donne comme explication à la volonté gouvernementale de maximiser $P$, le fait que le pouvoir de négociation est une fonction croissante de $P$. On doit y ajouter l'idée que l'incertitude liée au futur est une bonne raison de maximiser le $P$ présent: plus $P$ est élevé, plus, toutes choses égales par ailleurs, il a de chance de l'être dans le futur.

Bien entendu, la valorisation des $P$ futurs dépend de l'horizon du gouvernement. Si on accepte l'idée de Frey (1978, p. 206) selon laquelle l'horizon du gouvernement s'étend audelà de la prochaine élection ${ }^{7}$, alors le gouvernement a d'autant plus intérêt à maximiser $P$ à chaque période. La maximisation de $P$ permet d'appréhender un objectif autre que le maintien au pouvoir. La maximisation de $P$ sur une période permet à l'homme politique de viser un second objectif à la période suivante, à savoir : la politique exercée pour ellemême. Cette idée est aussi très proche de celle de Frey (1978, pp. 211-212), selon laquelle si le gouvernement possède un surplus de popularité, il est alors libre de poursuivre son idéologie.

Le temps passé au pouvoir finit donc par conditionner l'exercice du pourvoir. Lagadec (1997) fait l'hypothèse que plus un homme politique est au pouvoir depuis longtemps et plus son horizon personnel est lointain (plus il englobe de cycles électoraux). Cela signifie que l'homme politique pense d'autant plus au futur qu'il a un passé important en tant que gouvernant. Cette hypothèse est à relier à l'observation de Gallego (1996, p. 634), selon laquelle, plus un gouvernant est au pouvoir depuis longtemps et plus il a de chance de le rester. Le dirigeant de l'opposition ayant un temps d'expérience au pouvoir nul, son horizon est minimal : jusqu'à la toute prochaine élection, mais pas au-delà.

On peut donc en déduire que plus un système politique est " éclaté », plus les alternances sont probables et plus l'horizon des hommes politiques est court. Plus cet horizon est court et plus les incitations à influencer l'économie de façon opportunistes sont fortes ${ }^{8}$.

Comment l'économie peut-elle être influencée à des fins politiques? La croissance économique (évaluée sous différentes formes) est depuis longtemps identifiée comme une des principales variables de soutien politique. Voir sur ce point, notamment: Stigler (1973), qui, pratiquant des tests sur les élections au Congrès américain, identifie la variable déterminante de la réélection comme la variation du revenu par tête; Frey (1978), qui testant des fonctions de popularité sur trois pays (États-Unis, Royaume-Uni, 
Allemagne) isole trois variables significatives de soutien politique: la variation de la croissance du revenu disponible, le chômage et le taux d'inflation; Chappell Jr (1990), qui analysant conjointement les votes et les taux d'approbation pour les présidents américains, conclut que, tant les électeurs que les sondés évaluent la performance économique à travers l'inflation, le chômage (faiblement) et la croissance économique. Ainsi, plus la croissance s'améliorera et plus le gouvernant aura de chances d'être réélu. Or, la politique budgétaire est le premier instrument de stimulation de la croissance (indépendance des banques centrales, neutralisant la politique monétaire comme outil de soutien politique).

Dès lors que la vie politique est démocratique, rythmée par des élections, les politiciens au pouvoir sont donc tentés d'utiliser l'arme budgétaire à des fins électorales, c'est-à-dire essentiellement avant les élections. Voir sur la question de l'impact des cycles politiques Rogoff (1990) et pour un panorama des vérifications empiriques, voir Drazen (2000, chapitre 5, par exemple). On peut également noter que Shi et Svensson (2000) observent enfin que le cycle politico-économique est plus fort dans les pays en développement dans la mesure où la transparence y est plus faible et la manipulation plus facile. D'autres travaux plus récents décrivent le cycle plus finement en séparant l'activité législative en faveur des grands groupes (lois) et en faveur d'intérêts spécifiques (décrets) et en distinguant chronologiquement les pics de ces activités respectives à l'intérieur d'un cycle (Padovano et Petrarca, 2012 ; Padovano and Gavoille, 2013).

\subsubsection{Les manifestations de l'effet de cliquet}

La Nouvelle-Calédonie est très loin d'échapper à cette problématique. Et cela d'autant plus que l'éclatement du paysage politique (à la fois chez les indépendantistes et les nonindépendantistes) et les alternances successives récentes créent un contexte propice à raccourcir l'horizon des hommes politiques, ce qui incite aux comportements propres aux cycles politico-économiques.

29 La conséquence est qu'en cas de bonne surprise (recettes fiscales en hausse), la tentation est d'utiliser cette manne supplémentaire pour stimuler l'économie à court terme, ce qui présente deux inconvénients :

30 - Absence de constitution d'excédents pour les années difficiles, pour lesquelles une politique contracyclique ne sera donc pas possible;

31 - Augmentation des dépenses de fonctionnement (soit directement, notamment avec le recrutement de fonctionnaires supplémentaires, soit indirectement par des dépenses d'équipement qui nécessiteront ensuite de nouvelles dépenses de fonctionnement).

La Nouvelle-Calédonie échappe d'autant moins à cette problématique que la volatilité des cours du nickel se traduit, quand les cours sont hauts, par de bonnes surprises fiscales, qui annoncent tout aussi simplement les futures désillusions. La citation suivante illustre l'acuité de cette problématique :

33 "La Nouvelle-Calédonie [en tant que collectivité publique] connait fin 2009 une situation très dégradée de ses équilibres de fonctionnement qui fait suite à un très net tassement de ses recettes fiscales après une année exceptionnelle en 2007 qui a pu laisser augurer de la constitution d'excédents durables. L'accroissement des dépenses ( $+12 \%$ en moyenne annuelle depuis 2006) reste supérieur à l'évolution des recettes ( $+4 \%$ en moyenne annuelle sur la même période). Cette évolution à double vitesse crée un effet ciseau qui conduit à partir de 2008 à des déficits puisque le résultat net de 2008 s'établit à -1129 MF et s'amplifie en 2009 à -5822 MF. Ce résultat a un impact 
direct sur la capacité d'autofinancement (CAF) de la Nouvelle-Calédonie (...) (la capacité d'autofinancement devient même négative sur 2009, passant de 1357 MF en 2008 à -3927 MF). » (GNC, 2010, p. 20)

Comme nous l'avons évoqué (point 1.1.1.), le budget de répartition de la NouvelleCalédonie fait « redescendre » les recettes au niveau des autres collectivités. En période faste, chacun profite de rentrées fiscales élevées; dans les périodes plus difficiles, les problèmes (re)surgissent et ramènent notamment à des questions plus structurelles. Ces difficultés, du fait du budget de répartition, retombent donc également en « cascade » sur les autres collectivités. Le discours du Président de la province Sud du 25 mars 2010, bien que focalisé sur la clé de répartition, illustre clairement cette problématique ${ }^{9}$.

Les années fiscalement fastes se traduisent donc, presque toujours et partout, par des dépenses de fonctionnement supplémentaires, qui placent ensuite les collectivités dans une situation difficile lorsque les recettes diminuent. Dans le cas de la NouvelleCalédonie, ne peut-on pas considérer que ce comportement maximise à long terme la probabilité d'apparition d'une forme de malédiction des ressources? En effet, si on profite peu des meilleures années pour structurer l'économie, c'est-à-dire si la rente nickel est dilapidée à court terme, dans une perspective de croissance, mais pas de développement, que reste-t-il réellement à la fin?

\section{Encadré $\mathrm{n}^{\circ} 1$. la malédiction des ressources naturelles}

Encadré ${ }^{\circ} 1$ : la malédiction des ressources naturelles

La malédiction des ressources signifie que les pays riches en ressources se retrouvent « appauvris » par cette richesse. En 2001, Sachs et Warner ont mis en évidence que "presque sans exception, la croissance économique des pays dotés d'abondantes ressources naturelles a stagné depuis le début des années 70 ", constituant ainsi ce qu'ils nomment donc la malédiction des ressources. Les richesses naturelles de ces pays profitent donc à d'autres acteurs et avant tout aux firmes qui exploitent la ressource. Dans le Pacifique, l'exemple typique de malédiction des ressources correspond à la Papouasie Nouvelle-Guinée puisque ce pays très riche en ressources variées (notamment minérales) est également notoirement " pillé » par les FMN et dispose d'un IDH extrêmement faible, ce qui en fait un cas emblématique de malédiction des ressources, correspondant à plusieurs des causalités exposées par Frankel (2010) pour expliquer ce phénomène.

Frankel recense ainsi les causes pour lesquelles les pays riches en ressources se développent moins rapidement que ceux dépourvus de ressources naturelles Parmi ces causes: les tendances de long terme dans les prix internationaux, la volatilité des prix internationaux des ressources, les guerres civiles, la faiblesse des institutions ou encore le syndrome néerlandais.

Sur la question précise des institutions, Cabrales et Hauk (2010) montrent que pour les pays aux institutions politiques défaillantes, le capital humain est négativement corrélé à la valeur de leurs ressources naturelles (et inversement quand les institutions nationales sont de qualité). Le modèle présenté est de type compétition électorale mais avec une originalité : les électeurs sont supposés avoir un contrôle sur les politiques non seulement par le vote mais aussi par leur capacité à déclencher une révolution. Pour les pays aux institutions fragiles, les auteurs expriment ainsi également les conditions théoriques sous lesquelles de nouvelles découvertes de ressources naturelles devraient déboucher sur des révolutions politiques.

Dans le cas calédonien, le pacte de stabilité fiscale lui-même peut être vu comme une autre manifestation de la malédiction des ressources.

Le code des impôts calédonien accorde aux entreprises qui investissent dans de nouvelles usines, c'est-à-dire essentiellement les firmes multinationales minières (FMN), les avantages suivants: exonération temporaire (i) d'IS 35, (ii) de la contribution des patentes, y compris les centimes additionnels, (iii) de la contribution foncière, y compris les centimes additionnels, (iv) de la taxe générale sur les services affectant les prestations de services concourant à la construction du complexe - y compris les services de restauration collective et l'entretien des installations d'hébergement des personnels et à l'exclusion de certaines prestations réalisées par des membres des professions juridiques, judiciaires, comptables ou d'audit, $(v)$ des droits d'enregistrement, l'exonération portant sur les droits de mutation liés à l'acquisition d'éléments de l'actif d'exploitation et (vi) de la taxe hypothécaire liée aux opérations concourant à l'acquisition de biens immobiliers d'exploitation. Avant cette période d'exploitation, c'est-à-dire pendant la phase de 
construction, les mêmes avantages fiscaux s'appliquent, auxquels s'ajoute une exonération générale des droits d'importation. De plus, aucun nouvel impôt, créé postérieurement à leur agrément fiscal, ne peut être opposé aux entreprises agréées, sur l'ensemble de la durée d'application de l'agrément (d'où le nom de "pacte de stabilité fiscale ", alors qu'il s'agit en fait d'une exonération quasi-totale d'impôts).

Les avantages sont accordés pour une période maximale de quinze ans à compter de la date d'ouverture du premier exercice de mise en production commerciale (et dès lors que la production atteint $80 \%$ du nominal), majorée d'une durée de cinq ans pendant laquelle les avantages fiscaux sont réduits de moitié (si les projets d'investissement utilisent un procédé technologique permettant la mise en œuvre de minerais non encore traités en Nouvelle-Calédonie - cas de l'usine dite "du Sud", construite à Goro par Vale, qui valorisera des latérites alors que la seule usine actuellement en service en NouvelleCalédonie ne traite que des saprolites - ou s'ils sont implantés dans des zones géographiques dépourvues des infrastructure nécessaires à leur réalisation ou hors bassin d'emplois - cas de l'usine dite « du Nord », construite à Voh par Xstrata).

Ces exonérations ont été octroyées pour faire venir des industriels en Nouvelle-Calédonie, à un moment où les cours étaient très bas: les projections économiques de l'époque tablaient sur des cours de l'ordre de 7 dollars la livre de nickel (à titre ce comparaison : le cours moyen était, sur la période 2010-2011, de 9,5 USD/lb). Mais ces exonérations peuvent aussi devenir une véritable aubaine pour les investisseurs, si les cours restent sensiblement plus élevés.

40 Certes, le code des impôts prévoit certaines dispositions mettant fin prématurément au dispositif incitatif, mais ces dispositions renvoient à un calcul de la rentabilité de l'investissement s'appuyant sur un taux d'actualisation à fixer par la décision d'agrément fiscal. En pratique, pour les deux usines en construction, ce taux a été fixé à un niveau si élevé que, même si les cours sont au double de ce qui était prévu initialement par les industriels, le dispositif se prolongera effectivement jusqu'au terme des $15+5$ ans. De plus, le point de départ de ce délai est l'année où la production commercialisée atteint $80 \%$ de l'objectif : l'usine du Sud est ainsi quasi achevée depuis 2009, mais ne dépassera ce seuil, au mieux, qu'en 2012 soit 2013. Tant pour cette usine que pour l'usine du Nord, la Nouvelle-Calédonie est donc quasi-certaine de ne percevoir aucune fiscalité jusqu'en 2027 voire 2028.

41 C'est finalement une situation aberrante, sachant que la ressource a été attribuée gratuitement aux FMN et que les cours peuvent être favorables (c'est le cas depuis 2005) voire très favorables (2007).

42 A contrario, pour une revue des meilleurs instruments de taxation des rentes minières, on peut se référer à Garnaut (2010). L'auteur étudie les instruments fiscaux applicables à une rente minière - au nombre de six - et leurs possibles combinaisons en fonction des objectifs de stabilité, de neutralité de la taxe et de maximisation du rendement fiscal - ce dernier critère ayant été totalement abandonné en Nouvelle-Calédonie au profit de l'objectif d'attractivité.

La richesse en ressources naturelles de la NC, loin d'être un bienfait sans contrepartie, fait que ce territoire vérifie non seulement une forte vulnérabilité (avec 95\% des exportations de produits du nickel, on peut s'interroger sur le rapport de force entre les opérateurs et le gouvernement, fut-il considéré comme un décideur bienveillant), mais également une 
forte volatilité de ses recettes minières et qu'il présente des facteurs de risque de malédiction de ressources (alors que la ressource touristique stagne - cf. encadré $\mathrm{n}^{\circ} 2$ ).

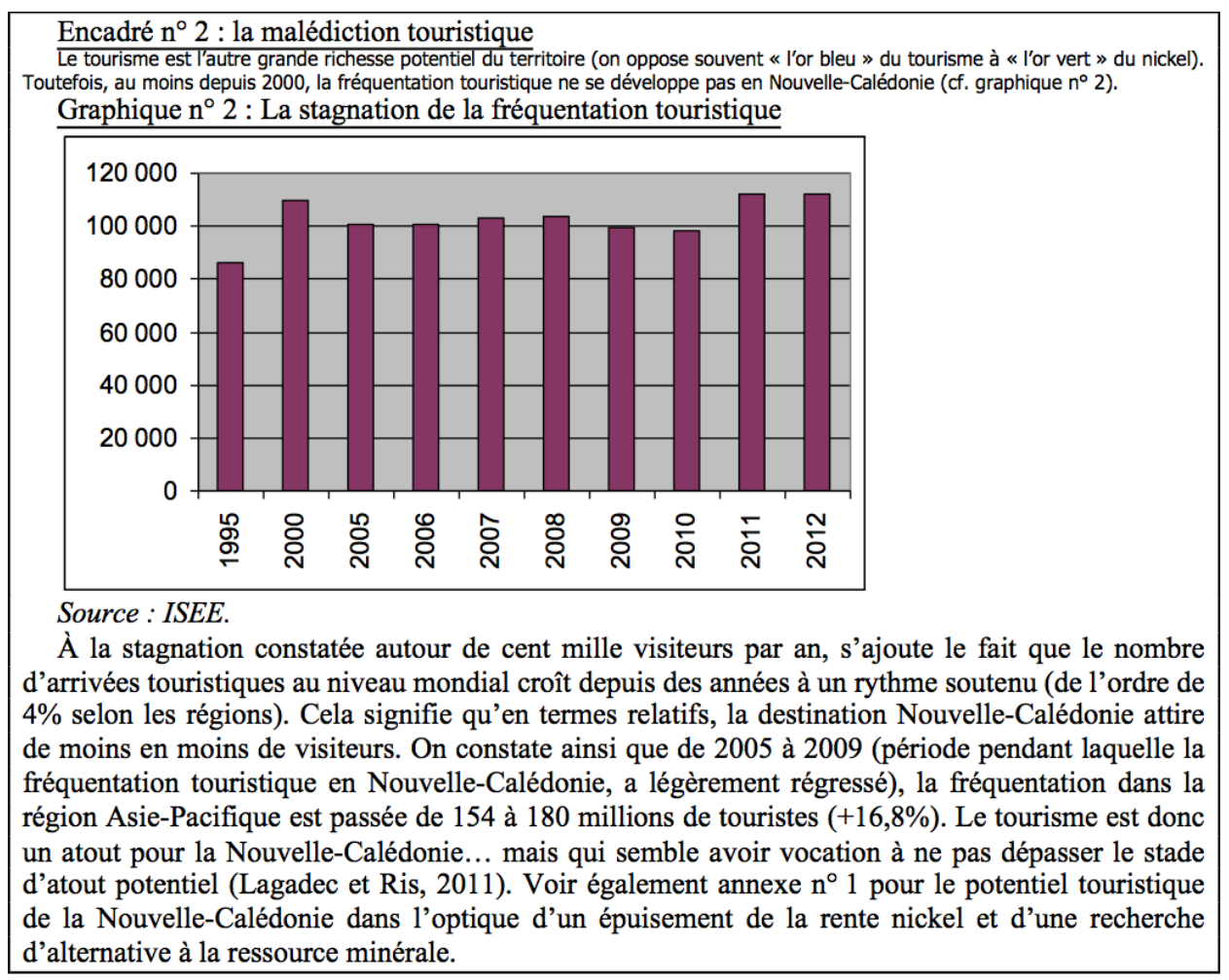

\section{Le fonds pour les générations futures}

Eu égard au cycle fiscal «bonnes surprises-désillusions », ne faut-il pas alors envisager d'« extraire » les recettes du nickel des comptes de la collectivité ? Cela signifierait qu'il faudrait neutraliser la variabilité des cours du nickel et donc des recettes de l'IS afférentes en affectant l'ensemble de ces ressources à un fonds pour les générations futures. Ainsi, en période de cours élevés on éviterait les effets de cliquet liés à l'augmentation des dépenses publiques (recrutement de fonctionnaires, etc.). Ce système ne pourrait a priori avoir de portée que pour l'IS 35 (cf. développements précédents).

\subsection{L'existant et les propositions}

\subsubsection{Le problème des permis de recherche et concessions}

Dès lors qu'une société obtient un permis de recherche, l'attribution d'une concession d'exploitation est de droit, dès lors que la présence d'une ressource exploitable a été démontrée. Le principe, c'est que les mineurs conduisent à leurs frais les travaux d'exploration et ont donc besoin de la garantie de pouvoir ensuite exploiter. Cette logique est héritée du code minier métropolitain. Elle est en vigueur en Nouvelle-Calédonie depuis plus de cent ans, et a été reconduite lors de l'adoption du code minier de la Nouvelle-Calédonie, celui-ci ayant suivi le modèle du code métropolitain.

Cette logique suppose l'existence d'un aléa, si bien qu'elle est bien moins pertinente pour les zones sur lesquelles on dispose déjà d'une information sur la richesse du sous-sol : par exemple, sur Prony, des forages avaient déjà été réalisés dans les années 1950. Le seul 
« risque » que prend l'entreprise effectuant des recherches concerne le coût des forages. Ensuite, une fois la concession obtenue elle est très difficile à retirer même en cas de nonexploitation, ce qui amène à un patrimoine minier en grande partie gelé ${ }^{10}$. Outre le pacte de stabilité fiscale, c'est l'ensemble de la réglementation qui est donc favorable aux firmes minières et métallurgiques, sans que l'intérêt du pays ou de ses populations ait été véritablement mis en avant.

\subsubsection{Redevance d'extraction, fonds de secours et fonds pour les générations futures}

47 La quasi-totalité des pays miniers appliquent un système similaire aux redevances d'extraction, sous le nom de royalties. Sauf la France, et donc sauf la Nouvelle-Calédonie. Ce système est plus adapté lorsque le coût d'exploitation est faible par rapport au prix de vente. Le pourcentage de royalties appliqué par les pays pétroliers dépasse par exemple souvent $50 \%$. Cette lacune du droit minier français est apparue publiquement avec la récente découverte de pétrole en Guyane.

En Nouvelle-Calédonie, les projets de redevance minière sont récurrents et sont le plus souvent reliés à la question de la création d'un fonds pour les générations futures. Il est ici important de noter que quelle que soit la forme qu'on lui envisage, un tel fonds pour les générations futures ne doit être confondu ni avec le fonds de concours pour le soutien conjoncturel du secteur minier, ni avec le « fonds nickel »"

Le premier est un fonds de concours créé par délibération n 502 du 17 août 1994 Alimenté par $15 \%$ de l'IS 35 versé par les sociétés minières, il avait pour objet de favoriser le maintien des emplois liés à la mine lorsque l'activité minière traverse une situation de crise (situation déclarée par arrêté du gouvernement). L'organisation et les modalités de fonctionnement du fonds avaient été définies par la délibération $\mathrm{n}^{\circ} 161$ du 29 décembre 1998 qui prévoyait deux axes d'aides pour les entreprises qui se trouveraient en difficulté : la prise en charge partielle des cotisations sociales patronales pour l'année 1999, et le financement partiel d'opérations de réhabilitation de certains sites miniers. En 2002, la délibération $n^{\circ} 290$ avait étendu la possibilité de prise en charge des cotisations sociales au-delà de l'année 1999, et avait fixé des critères d'éligibilité des entreprises.

Le second est un établissement public administratif créé par délibération du 18 mars 2009, ayant un double objet : d'une part, comme le fonds conjoncturel, aider la profession à passer les périodes de retournement des cours; d'autre part, financer la réhabilitation environnementale de zones dégradées par l'activité minière.

51 La délibération $n^{\circ} 49$ du 30 décembre 2004 a plafonné le fonds de concours à 1,5 milliard de francs CFP. L'art. 3 de la délibération stipule ainsi : «Au B de l'article 2 de la délibération modifiée $n^{\circ} 161$ du 29 décembre 1998 susvisée, les termes : "dans la limite de 250 millions de francs CFP par entreprise et par an." sont remplacés par les termes: "dans la limite d'un plafond de 1,5 milliard de francs pour le fonds." " En conséquence, le fonds ne sera plus réalimenté que lorsque du fait de son utilisation, il aura chuté en deçà de ce seuil. Cette décision de plafonnement est un cas d'école de décision ne préparant pas l'avenir. En effet, si on n'alimente pas le fonds pendant une période de croissance et de cours élevés, quand le fera-t-on? Cette décision avait d'ailleurs fait l'objet d'âpres débat au congrès et de critiques récurrentes ${ }^{12}$. Il faut comprendre cette décision à la lumière des développements précédents sur les cycles politiques et l'horizon court des politiciens, d'autant plus court que les alternances politiques sont récentes (voir supra point 1.2.1.) ${ }^{13}$. 
52 Le projet de créer un fonds pour les générations futures figurait dans la Déclaration de politique générale d'Harold Martin, Président du gouvernement calédonien en janvier 2008 : «Il est proposé de faire mieux et de prévoir que nos descendants puissent aussi profiter de notre richesse actuelle, en créant un fonds pour les générations futures. Cette idée n'est pas originale, les pays producteurs de pétrole ou de gaz l'ont mise en ceuvre. Ce fonds pourrait prendre la forme d'une fondation, puisque l'État s'est engagé, lors du dernier Comité des signataires à rendre applicable en Nouvelle-Calédonie la législation nécessaire.

Quelle que soit sa forme juridique, le fonds doit disposer de ressources pérennes qui pourraient être une taxe ad valorem sur l'exploitation du minerai, ou une affectation d'une partie de l'impôt sur les sociétés métallurgiques, ou toute autre participation obligatoire ou volontaire. Ce fonds pourrait reprendre les actions $d u$ fonds conjoncturel minier, mais il doit essentiellement mener des actions qui ont un impact à long terme, sur l'environnement, sur les équipements structurants durables, ou sur les réserves des organismes de protection sociale pour les droits sociaux à venir. L'objet de ce fonds est d'investir pour l'avenir. Le code minier traduira dans la loi les orientations du schéma de mise en valeur des richesses minières. ».

Pour autant, le fonds pour les générations futures en est resté au stade de projet, puisque, bien qu'annoncé dans le schéma de mise en valeur des ressources minières, il n'a jamais été concrétisé.

Même si des pistes sont régulièrement évoquées, la difficulté réside dans l'outil d'abondement du fonds. En effet, la contrainte du pacte de stabilité fiscale empêche de nouveaux impôts sur les sociétés minières ; pour cette raison Harold Martin envisageait que l'IS 35 abonde directement le fonds.

C'est également pour cette raison que Philippe Gomès (réintroduisant le projet en tant que nouveau Président du gouvernement dans sa Déclaration de politique générale en août 2009) a pris le parti d'une redevance domaniale d'exploitation. Cet outil n'étant pas un impôt on pouvait considérer qu'il "échappait» au pacte de stabilité fiscale ${ }^{14}$. La déclaration de politique générale d'août 2009 exposait que le produit de cette redevance devait alimenter le Fonds nickel pour les générations futures : «La possibilité de création d'une redevance domaniale applicable à la tonne de minerai extraite, indexée sur le cours du Nickel, sera examinée. Le produit de cette redevance sera affecté au Fonds Nickel pour les générations futures, fonds qui sera également susceptible de recevoir, en période de forte croissance, les surplus budgétaires de la Nouvelle-Calédonie. En effet, de nombreux pays ont constitué, ces dernières décennies, des fonds souverains qui poursuivent des objectifs d'investissement à long terme et qui sont alimentés par des recettes tirées de l'exploitation des matières premières. La Nouvelle-Calédonie doit s'inspirer de ces exemples, notamment dans la perspective du retour au régime fiscal de droit commun pour les usines métallurgiques du Nord et du Sud.»

La Déclaration de politique générale d'août 2009 esquissait ainsi les contours d'un fonds pur les générations futures tel qu'il est pratiqué par la Norvège.

En 2013, le second gouvernement Martin a relancé le projet d'une réforme de la fiscalité minière, préparant une loi de pays instituant une contribution à l'exportation, censée alimenter le Fonds Nickel, avant qu'un fonds pour les générations futures (non créé par la loi) ne s'y substitue pour recevoir les produits de la taxe. Ce projet semble peu adapté aux besoins de financement (rendement prévu de l'ordre de 3 milliards de F. CFP par an, après démarrage de l'usine du Nord et de celle du Sud) et très fragile juridiquement puisque le 
choix d'une taxe et non d'une redevance d'extraction heurtera de front le pacte de stabilité fiscale.

\subsection{Le modèle norvégien}

\subsubsection{Les défis à relever} charge aux générations futures qui bénéficieront des prestations. Mais, cette option n'est pas sans risque. L'évolution de la pyramide des âges montre en effet que le taux de dépendance (c'est-à-dire le nombre de jeunes de moins de 20 ans et des seniors de plus de 60 ans, ramené au total des personnes en âge de travailler) va augmenter sensiblement passant de 1,1 aujourd'hui à 1,3 dans 20 ans. Autrement dit, les 16000 actifs supplémentaires que comptera la Nouvelle-Calédonie d'ici à 2033 auront à leur charge 50000 « dépendants » de plus (4000 jeunes âgés de moins de 20 ans et près de 46000 senior de plus de 60 ans). Un supplément de charge, même « raisonnable » dans l'absolu, pourrait s'avérer alors beaucoup plus problématique à financer dans ce contexte (sauf à se résoudre à augmenter considérablement les prélèvements obligatoires sur les actifs, mais au risque alors de les voir fuir).

64 Ce risque peut être partiellement évité en instituant un mécanisme de financement intergénérationnel par capitalisation.

\subsubsection{Un fonds souverain alimenté aussi par d'autres ressources que le nickel}

La seconde option consiste à abonder un fonds ad hoc de manière à ce que le produit des capitalisations successives finance les besoins futurs.

Ce fonds pourrait être abondé en prélevant, chaque année, une fraction du PIB (ou en lui affectant une partie des recettes fiscales avant répartition). Ces ressources seraient placées sur les marchés financiers à court terme (en diversifiant les risques); les intérêts nets des tirages seraient capitalisés. Les montants devant abonder ce fonds dépendent principalement des dépenses qu'il aurait à financer ainsi que du taux d'intérêt créditeur.

Ce fonds pourrait venir financer : 
1. La charge nette supportée par la Nouvelle-Calédonie au titre des transferts de compétence dans l'éducation secondaire ;

2. Les déficits annuels des régimes de retraite induits par la croissance du nombre d'ayant-droits dans l'hypothèse d'une stabilité (au niveau actuel) tant des taux de prélèvements pour le financement du risque vieillesse que de l'âge de départ en retraite.

Dans ce cas, les besoins de financement auxquels devrait répondre ce fonds seraient de l'ordre d'une quinzaine de milliards de F. CFP en 2020, de 35 milliards en 2025 et d'une soixantaine de milliards en 2030 (voir graphique $\mathrm{n}^{\circ}$ 3).

Graphique 3. Besoins de financement intergénérationnels

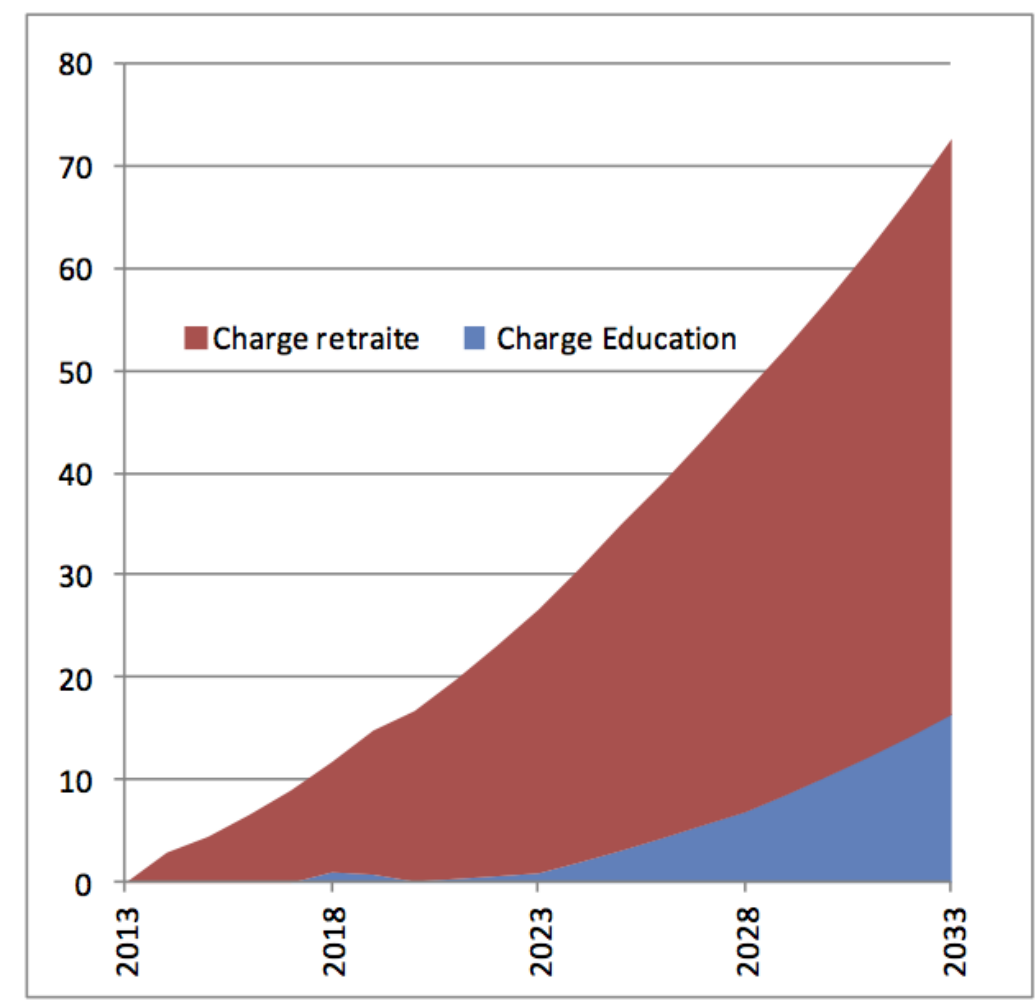

unité : milliards de F. CFP

Source : Sudrie (2011)

71 Cette charge pourrait être supportée amplement par un prélèvement annuel équivalent à $2 \%$ du PIB placé sur les marchés au taux de 3\% (ce qui représente un faible rendement comparé aux performances des fonds souverains gérés de manière dynamique comme ceux de Dubaï ou d'Abu Dhabi). Le profil du fonds est représenté sur le graphique $\mathrm{n}^{\circ} 4$, courbe bleue. Le fonds devrait croître jusqu'en 2025 pour atteindre un maximum de 220 milliards de F. CFP (soit un peu moins de $30 \%$ du PIB actuel). Passé cette date, le financement des besoins - essentiellement des retraites - suppose une décapitalisation (tirage sur le capital). Le profil du fonds est sensible aux hypothèses concernant le taux d'intérêt. Un taux de rendement de $4 \%$ (courbe rouge): (i) reculerait de 2 à 3 ans la date où le fonds atteindra son plafond; (ii) augmenterait la valeur de ce plafond (250 milliards contre 220) et (iii) réduirait la décapitalisation. 
Graphique 4 : Profil du fonds de capitalisation

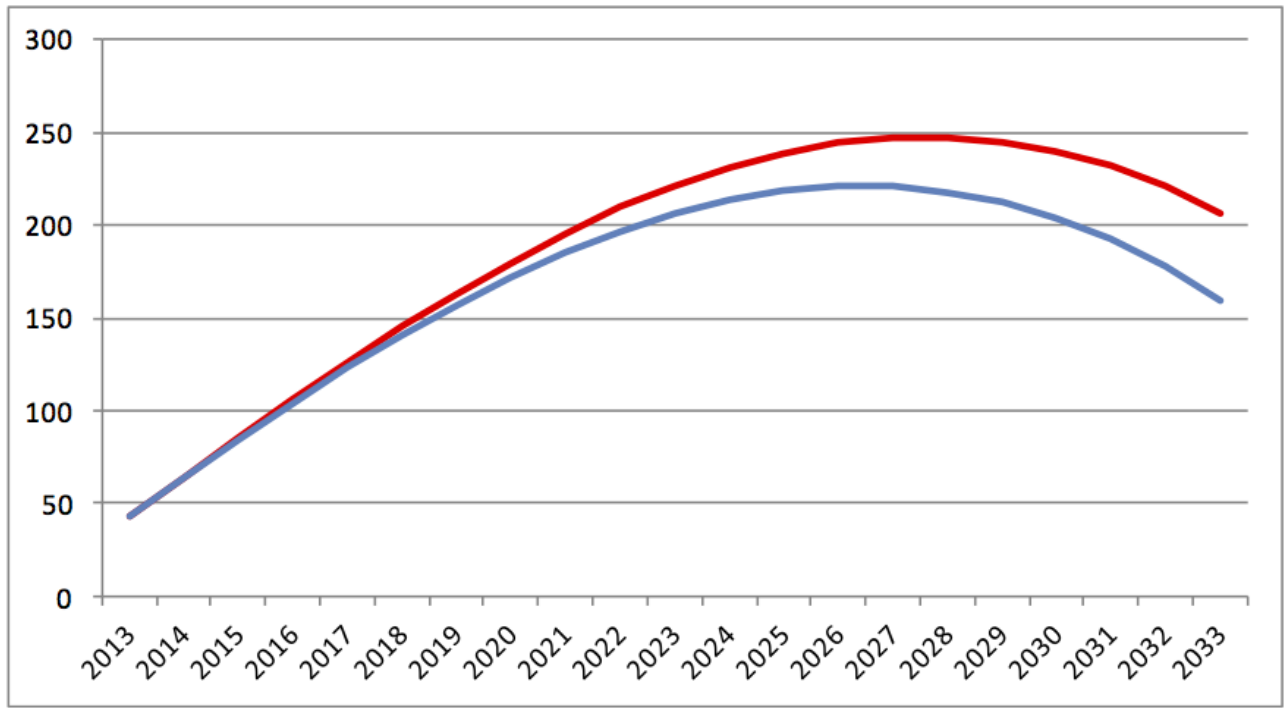

unité : milliards de F. CFP

Source : Sudrie (2011);

Le taux de prélèvement de $2 \%$ du PIB apparaît comme un minimum pour financer les charges à venir (sans empêcher néanmoins une décapitalisation à partir de 2030). Un taux de $1 \%$ (courbe rouge sur le graphique $n^{\circ} 5$ ) s'avère en effet insuffisant et conduirait à fermer le fonds (capitalisation nulle) dès 2025. Inversement, un taux de prélèvement plus ambitieux de $3 \%$ (courbe verte) empêcherait la décapitalisation à l'horizon de la projection (le fonds pouvant éventuellement financer alors d'autres charges que celles retenues dans cet exercice).

Graphique 5 : Profil du fonds de capitalisation selon les taux de prélèvement

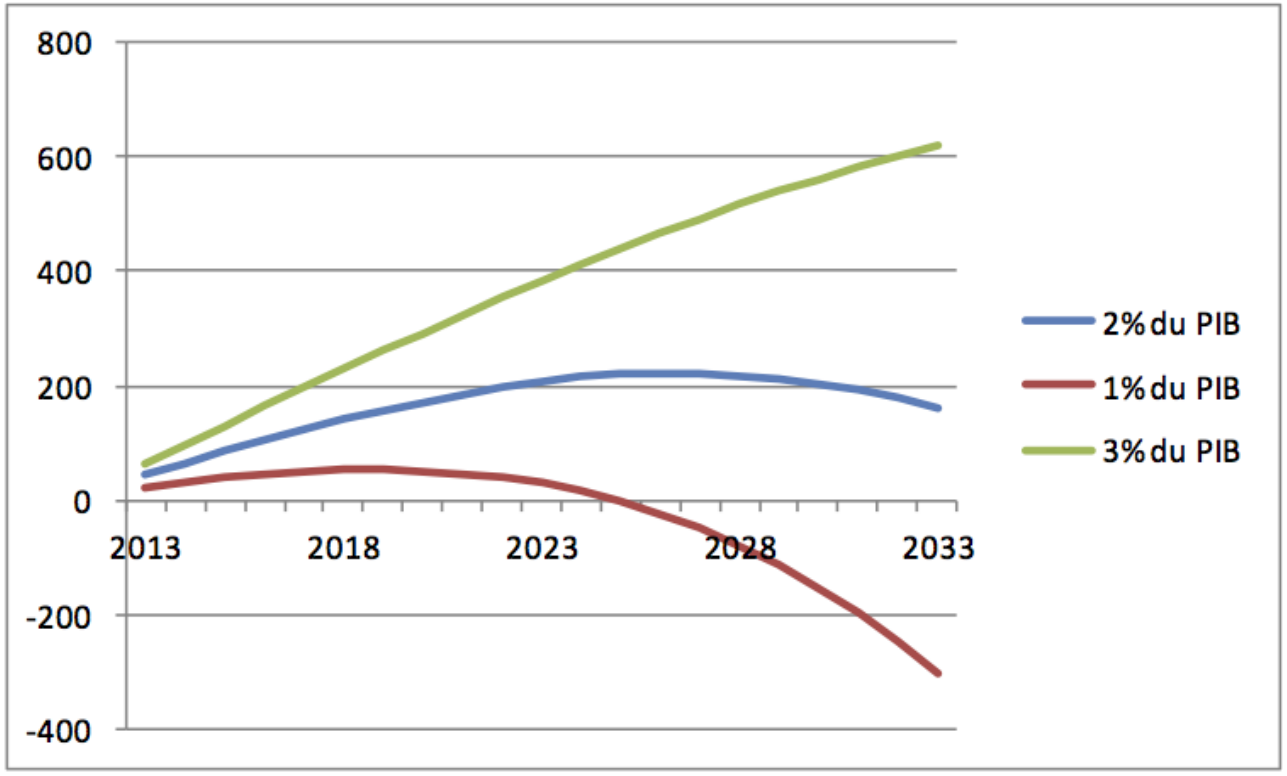

unité : milliards de F. CFP

Source : Sudrie (2011) 
Les recettes de l'IS 35 pourraient être utilement mobilisées pour alimenter le fonds. Mais, elles demeureront toujours insuffisantes pour financer les charges à venir énumérées cidessus. Comme le montre le graphique $n^{\circ} 6$, les recettes fiscales sur le nickel peuvent être estimées à moins de 15 milliards par an jusqu'en 2017 (et dans une hypothèse favorable de maintien des cours à $8 \mathrm{USD} / \mathrm{lb}$ ). Ces recettes représenteraient en moyenne le tiers du total des prélèvements annuels affectés au fonds. La fin du pacte fiscal (ici à dater de 2028) allégerait d'autant les prélèvements sur la valeur ajoutée hors nickel ${ }^{15}$; l'IS 35 contribuant à $50 \%$ environ au total des versements à l'horizon de la projection.

Graphique 6 : IS 35 et versements au fonds

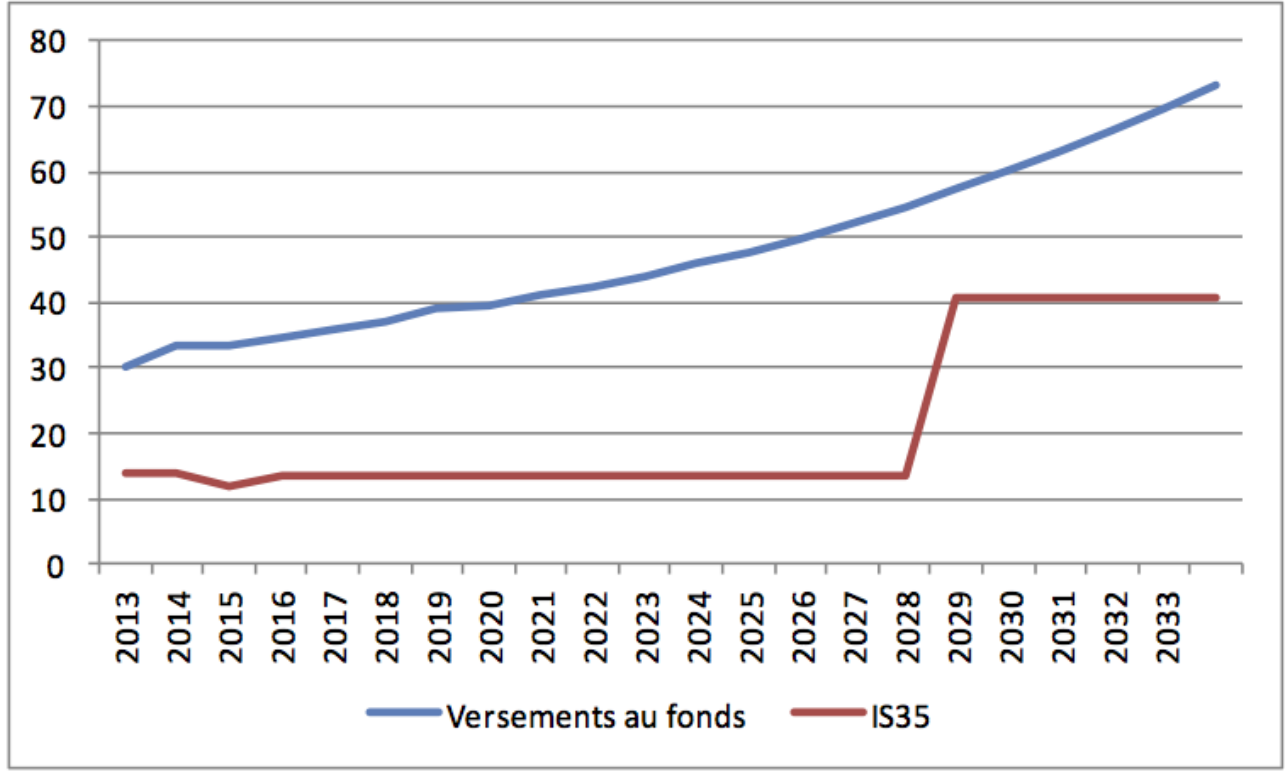

unité : milliards de F. CFP

Source : Sudrie (2011)

\section{Conclusion} politique calédonienne: souvent évoqué, jamais concrétisé. C'est pourtant le seul instrument qui s'inscrirait résolument dans une optique de développement durable et de prévention du syndrome de la malédiction des ressources. valeur des richesses minières de la NC stigmatise ainsi «Un contexte économique extrêmement favorable mais non durable ne participant pas au développement des générations futures »-GNC, 2009, p. 131).

Le pacte de stabilité fiscale, reflet lui-même de la malédiction des ressources, de la puissance des firmes métallurgiques et minières et de leurs relais politiques, complique l'abondement d'un tel fonds. La problématique des cycles politiques, selon laquelle les gouvernants sont plus incités à dépenser les recettes publiques dans une optique immédiate plutôt qu'à préparer réellement l'avenir, est un autre point de blocage. 


\section{BIBLIOGRAPHIE}

Austen-Smith D., Wright J. R. 1992, "Competitive Lobbying for a Legislator's Vote", Social Choice and Welfare, 9, 229-257.

Breton A., 1981, "Representative Governments and the Formation of National and International Policies", Revue Économique, 2, Mars, 356-373.

Cabrales A., Hauk E., 2010, "The quality of political institutions and the curse of natural resources", The Economic Journal, à paraître.

Chappell Jr. H, W, 1990, "Economic Performance, Voting, and Political Support: A Unified Approach", The Review of Economics and Statistics, 313-320.

CTC (Chambre Territoriale des Comptes de la Nouvelle-Calédonie), 2008, Rapport d'observations définitives établi à la suite de l'examen de la gestion des recettes fiscales et douanières de la NouvelleCalédonie, 13 novembre, 74 pages.

Drazen, A., 2000, "The political business cycle after 25 years", Working Paper.

Farvaque E., Paty S., 2007, Économie de la Démocratie, de Bock, 116 pages.

Frankel J., 2010, "The natural resource curse: a Survey", NBER Working Papers, No. 15836.

Frey B. S., 1978, "Politico-Economic Models and Cycles", Journal of Political Economy, 9, 2, 203-220.

Frogier P., 2010, Déclaration d'orientation des actions prioritaires de la province, Assemblée de la Province Sud - Nouméa, Jeudi 25 mars 2010, 15 pages.

Gallego M. E., 1996, "Interest Groups, Government Turnover and Political Regimes: An Econometric Analysis", Canadian Journal of Economics, 24, Avril, 633-638.

Garnaut R., 2010, "Principles and practice of resource rent taxation", The Australian Economic Review, vol. 43, No. 4, December, 347-356.

Développement durable et territoires, Vol. 4, $n^{\circ} 3$ | Octobre 2013 
Gay J-C., Saïssi O., 2010, « Pourquoi rechercher des alternatives à la ressource minérale », in La Nouvelle-Calédonie et le développement minier : Trajectoires passées, présentes et futures, Projet de recherche déposé auprès du CNRT Nickel - Nouméa, section 5.3.2.3., 311 pages. [Projet non retenu par le CNRT « Nickel et son environnement »]

GNC (Gouvernement de la Nouvelle-Calédonie), 2009, Le schéma de mise en valeur des richesses minières de la Nouvelle-Calédonie, mars, 203 pages.

GNC (Gouvernement de la Nouvelle-Calédonie), 2010, Rapport sur les orientations budgétaires 2011-2013, août, 38 pages.

Gomès G., 2009, Déclaration de politique générale devant le congrès de la Nouvelle-Calédonie, 31 août.

Lagadec G., 1997, Des intégrations régionales au libre-échange généralisé : les enseignements d'un nouveau modèle de protection endogène, thèse de doctorat, Lille 1,252 pages.

Lagadec G., Ris C., 2011, « La Nouvelle-Calédonie entre fragilités sociales et réformes économiques ", INFORMATIONS et Commentaires - Le développement en questions, ISMEA, 2011, Mars, 14 pages.

Martin H., 2008, Déclaration de politique générale devant le congrès de la Nouvelle-Calédonie, 4 janvier. NC 2025, 2009, Schéma d'Aménagement et de Développement de la Nouvelle-Calédonie, Diagnostic et enjeux, septembre.

Padovano F., Petrarca I., 2012, "A Theory of Cyclical Production of Laws and Decrees", Working Paper 2012-09, Condorcet Center for Political Economy, University of Rennes 1, 24 pp.

Padovano F., Gavoille N., 2013, "The Dual Political Legislation Cycle in France", Working Paper 2013-02, Condorcet Center for Political Economy, University of Rennes 1, April, 32 pp.

Rogoff K., 1990, "Equilibrium Political Budget Cycles", American Economic Review, 80, 21-36.

Shi, M., Svensson J., 2000, "Political business cycles in developed and developing countries", Working paper, The World Bank, February.

Stigler G., J., 1973, "General Economic Conditions and National Elections", American Economic Association Papers and Proceedings, 63, 2, p. 160-167.

Sudrie O., 2011, « Scénarios de développement de la Nouvelle-Calédonie à l'horizon 2025 », Document de travail DME, Gouvernement de la Nouvelle-Calédonie, Direction générale des services, Service de l'aménagement et de la planification, décembre.

Syndex, 2005, Nouvelle-Calédonie Nickel 2010 : Une nouvelle aire industrielle, Colloque international, USOENC, 7 et 8 juillet 2005, 189 pages.

Ward H., 1993, "Game Theory and the Politics of the Global Commons", Journal of Conflict Resolution, 37, 2, 203-235.

\section{ANNEXES}

Annexe 1. Pourquoi rechercher des alternatives à la ressource minérale

(Gay et Saissi, 2010).

Faire reposer le développement d'un pays sur un secteur mono exportateur présente des difficultés assez intuitives : soumissions aux fluctuations des cours internationaux, sans 
possibilité de « lisser » les périodes de cours bas par d'autres ressources à l'activité non synchronisée, maximisation des effets d'un éventuel syndrome néerlandais...

En ce sens, développer une autre ressource est conforme au principe de précaution. Dans le cas de la Nouvelle-Calédonie, il s'agirait de développer l'autre secteur pour lequel le territoire possède un avantage comparatif naturel, à savoir le tourisme.

Historiquement symbole de l'essor économique calédonien, le nickel pourrait bien vaciller de son piédestal sous l'influence de plusieurs facteurs qui vont connaître dans un avenir plus ou moins proche un essor important :

Des préoccupations en matière de santé publique : à l'instar de nombreux composants chimiques, le nickel est toxique à hautes et très hautes doses, situation que l'on connait à proximité des sites d'extraction ou de traitement du minerai ${ }^{16}$. Les conséquences sur les populations se traduisent par des dermo, cardio et pneumo pathologies. Des hypothèses de cancérogènèses, de pathologies pédiatriques, de décès prématurés des nourrissons sont également formulées sans, pour l'instant avoir été confirmées par des analyses épidémiologiques. Cependant, compte tenu des expériences, la formulation d'une hypothèse assimilant le nickel à l'amiante en termes de risques sur les populations n'est pas hérétique en soi. Dès lors, sous la pression de l'opinion publique, des instances de régulation, des mesures de protection peuvent être élaborées et restreindre l'industrie ou l'utilisation du nickel. Par exemple, telle peut être comprise la décision des autorités finlandaises visant à proscrire l'utilisation des pièces de 1, 2 et 5 centimes d'euros en raison de leur teneur en nickel.

Une évolution des technologies et matériaux utilisés dans l'univers industriel : Dans le cas du nickel calédonien, on considère traditionnellement que l'importance des réserves dispense de la nécessité de rechercher d'autres ressources à moyen et long terme (comme c'est le cas pour, au moins, certains pays pétroliers). Pourtant, si les réserves de nickel en Nouvelle-Calédonie, sont immenses on ne peut négliger le risque que le nickel lui-même perde son attrait industriel.

Depuis des décennies, le nickel est ajouté à des métaux afin de rendre les alliages obtenus inoxydables. Cette particularité chimique est à l'origine du fort développement de l'activité minière. Les recherches, menées notamment dans le domaine des composites de carbones, tendent, peu à peu, à faire apparaitre des substituts aux alliages à base de nickel dans la construction d'aéronefs, de navires voire d'automobiles. Ainsi le nouvel avion Boeing 787 Dreamliner est-il le premier aéronef entièrement réalisé en composites de carbone à l'exception des réacteurs. Les composites de carbone et les bio composites en voie de réalisation présentent plusieurs avantages par rapport au nickel : ils sont plus légers et plus résistants. De plus, leur obtention ne nécessite pas d'extraction minière. Actuellement, le seul inconvénient, qu'ils présentent, réside dans le coût économique d'obtention. Cependant, avec le développement technologique et la croissance des volumes fabriqués, une diminution du coût économique va être induite ce qui devrait fortement limiter l'opportunité des alliages à base de nickel.

- Une source de richesse qui se tarit : L'activité minière, en Nouvelle-Calédonie, a pris son essor avec la présence d'un seul acteur : la Société Le Nickel (SLN). Avec l'orée des années 2000, deux nouveaux acteurs sont apparus (cf. partie 03.3) :

L'Usine du nord dénommée Koniambo Nickel SAS soutenue notamment par la SMSP ;

L'Usine du sud dénommée Vale Inco Goro Nickel. 
Voués à réaliser une production identique, le nickel, ces acteurs vont naturellement entrer en concurrence et engendrer une diminution du prix de ce minerai sur le marché mondial en raison d'une augmentation, à terme, de l'offre pour une demande vouée à décroître. De plus, à la faveur de la mise en place de stratégies financières, certains acteurs peuvent être enclins à commercialiser leur production à prix réduit, à des filiales situées à l'étranger, leur permettant de réaliser ainsi des profits qui échappent à la fiscalité locale, diminuant ainsi les ressources perçues par le gouvernement calédonien.

Un déséquilibre en termes d'aménagement du territoire calédonien : L'activité du nickel est inégalement répartie (cf. carte suivante).

Cette disparité géographique induit une hétérogénéité des zones d'activités économiques, laissant des territoires pourtant habités quasiment inertes. Ainsi, à la lecture de la carte $n$ ${ }^{\circ} 1$, on constate : une forte localisation de la population, particulièrement d'origine européenne, en province Sud et une localisation importante de population d'origine mélanésienne dans le Nord-Est et sur les îles Loyauté alors que l'activité économique y est très réduite.

Carte 1.: La localisation des exploitations minières

Répartition géographique de l'activité du nickel en Nouvelle Calédonie

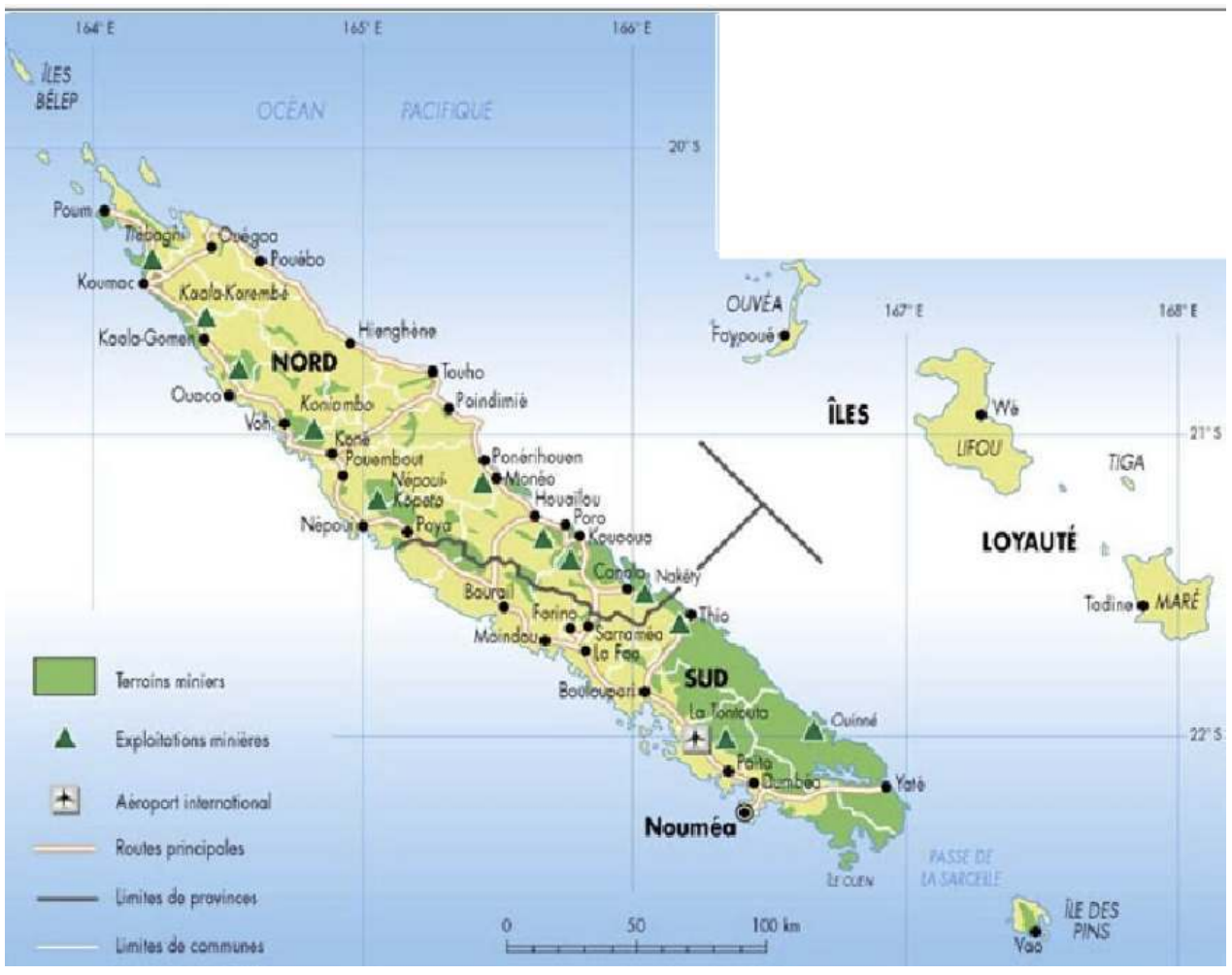

Carte 2. La Répartition de la population calédonienne 


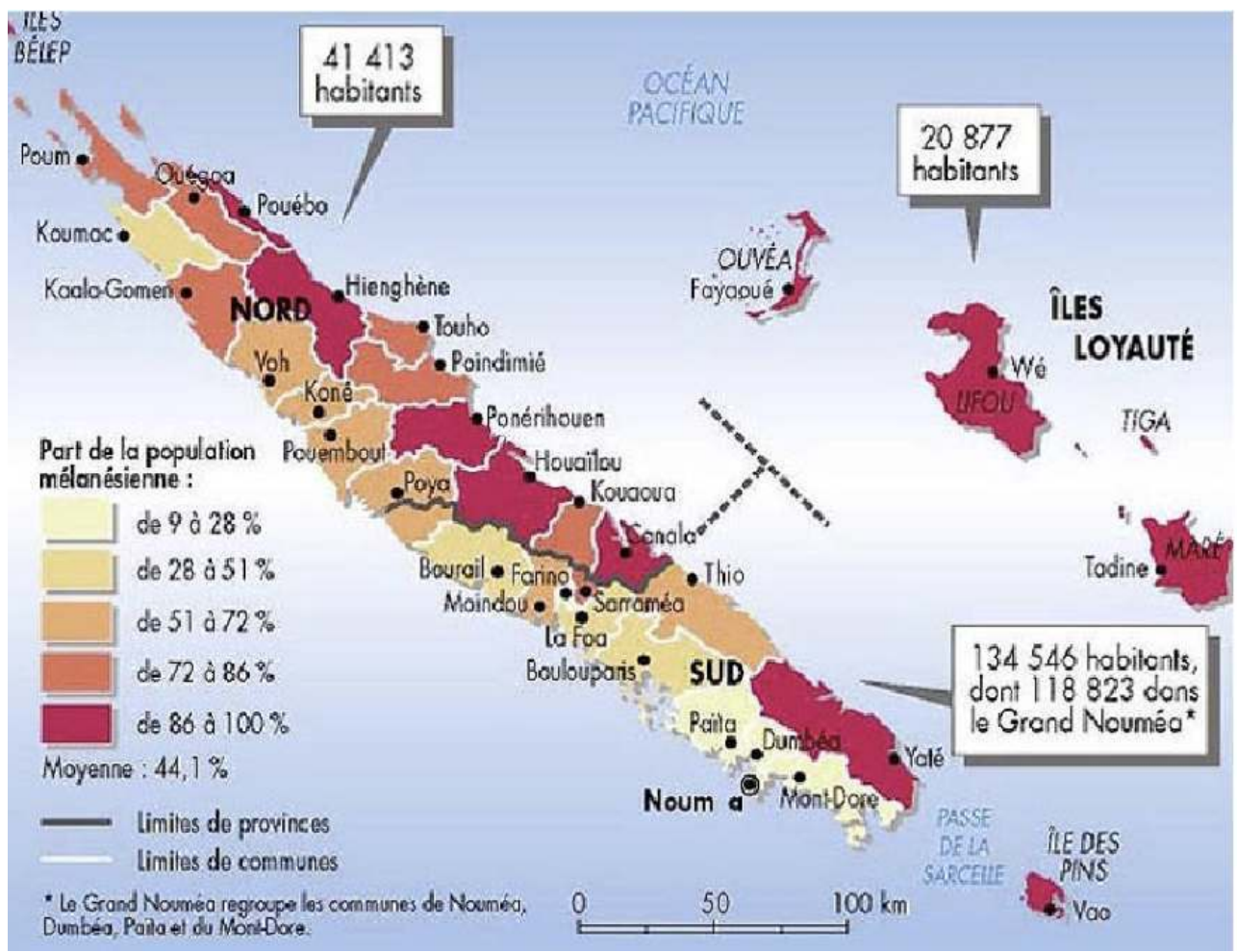

La très faible corrélation, dans le nord et sur les îles, entre la répartition de la population et l'activité économique est de nature à générer deux conséquences :

Un exode des populations vers les centres économiques saturant potentiellement des infrastructures qui ne sont pas adaptées à leur accueil ;

Une absence d'activités de populations qui vont alors être dépendantes des aides publiques et/ou des viatiques dispensés par des travailleurs migrants.

Impact sur l'environnement, remise en cause technologique, déséquilibrage économique, à défaut d'être remplacée, l'activité nickel doit tout au moins, être complétée. Eu égard, la localisation et la géographie de la Nouvelle Calédonie, les activités touristiques apparaissent comme un élément de réponse à la problématique posée.

Annexe 2. La répartition du budget 2012 de la Nouvelle-Calédonie (millions CFP) 


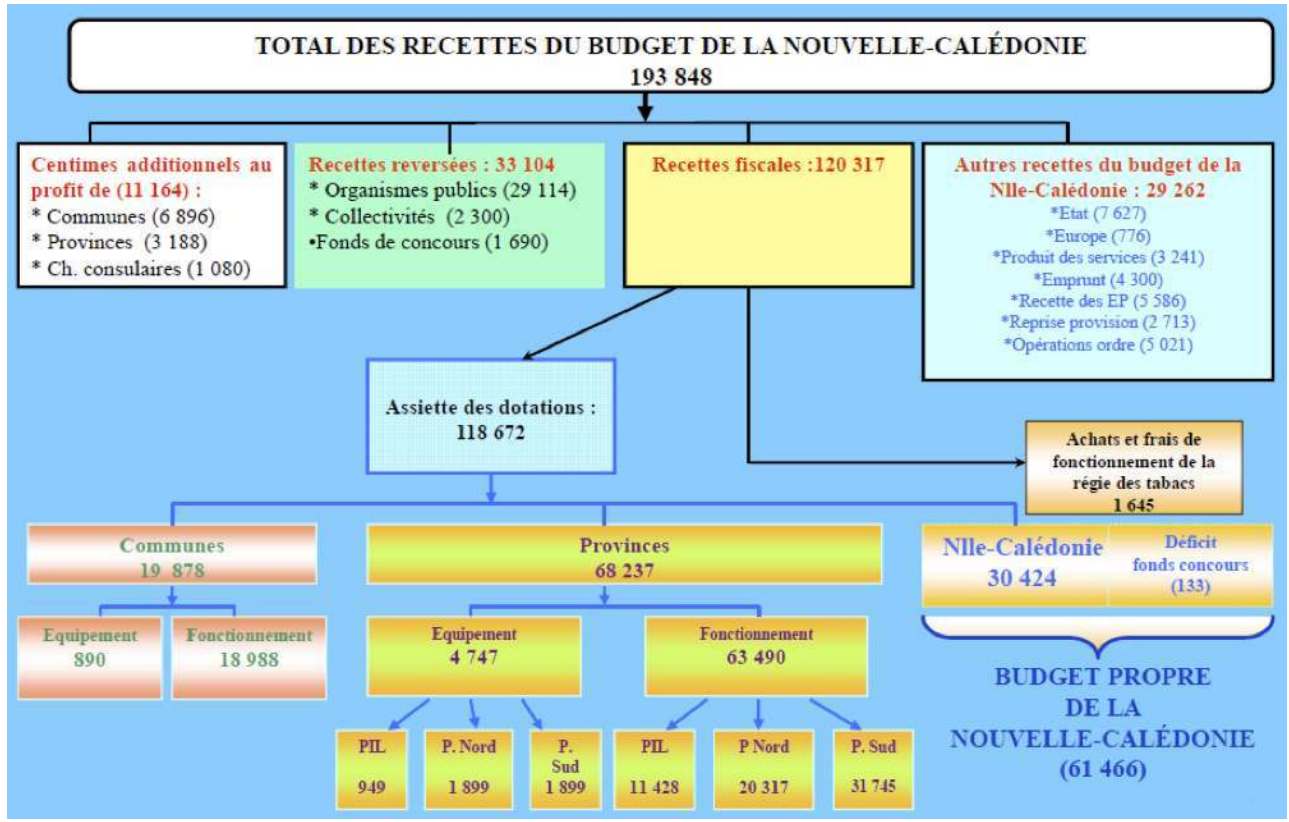

Source: Direction du Budget et des Affaires Financières de la NC.

\section{NOTES}

1. Ces $26 \%$ ne sont cependant pas une règle intangible, car on peut librement affecter certains impôts à des établissements ou services publics. Par exemple, le besoin de financement découlant de la création du minimum vieillesse ne va entrainer une augmentation d'impôt qu'à hauteur, exactement du financement nécessité, et non pas 4 fois plus, grâce au fait que cette augmentation d'impôt sera affectée à l'ASS, chargée du financement de ce dispositif.

2. Selon le schéma de mise en valeur des ressources minières de la $\mathrm{NC}$, «Aujourd'hui, les principales retombées fiscales du secteur de la mine et de la métallurgie sont perçues au travers de l'impôt sur les sociétés et les activités métallurgiques ou minières. Pour tenir compte des autres avantages dont elles bénéficient déjà par ailleurs, notamment en matière douanière, le taux de l'impôt sur les sociétés réclamé à ces entreprises est de $35 \%$, contre seulement $30 \%$ pour les autres sociétés. En outre, plusieurs taxes et redevances ont été instituées, visant spécifiquement l'activité minière et métallurgique : (i) la taxe pour déprédation de voirie, liée au roulage sur des routes classées, (ii) les droits sur les demandes d'autorisations personnelles minières et de titres miniers, (iii) la redevance ordinaire, sur les titres miniers détenus au titre de l'allocation d'un périmètre, (iv) la redevance supplémentaire annuelle, sur les titres miniers non exploités » (GNC 2009, pp. 131-132).

3. Le franc CFP est la monnaie ayant cours légal dans les territoires français du Pacifique. Le franc CFP dispose d'une parité fixe avec l'euro (1 euro égal 120 francs CFP).

4. Cette question (qui regroupe quatre idées : part dans le PIB, part dans l'emploi, relativisation des exportations, évasion des profits) est très vaste et mériterait une étude à part entière.

5. On peut modifier la fiscalité, mais si cela aggrave les charges d'une société ayant un agrément fiscal (Koniambo Nickel SAS et Vale Nouvelle-Calédonie SAS), celle-ci sera fondée à demander un remboursement. C'est valable pour toute modification de la fiscalité, et pas seulement de la fiscalité minière. Ce cas se produira avec la TGA, qui va entraîner un remboursement à KNS et Vale d'une partie de la TGA qu'elles vont payer auprès de leurs fournisseurs. 
6. Le cycle partisan signifie qu'une fois élu le gouvernant adoptera une politique quasiexclusivement tourné vers ses soutiens ou ce qu'il considère comme son électorat. Dans ce cadre, la manipulation de l'économie intervient après l'élection (avant dans le cycle opportuniste).

7. Cette hypothèse se retrouve d'ailleurs également chez Ward (1993, p. 230), puisque, si l'auteur estime que les hommes politiques ont souvent des horizons courts, il estime également que c'est la conscience, par ces hommes politiques, du caractère non infini de leurs carrières, qui restreint leur horizon. Or, la carrière d'un homme politique est supérieure à n'importe quel cycle électoral.

8. Selon la célèbre définition de James Freeman Clarke (écrivain américain, 1810-1888) : «Un politicien pense à la prochaine élection. L'homme d'État, à la prochaine génération. " (Cité par Farvaque et Paty, 2009).

9. Frogier, 2010, Déclaration d'orientation des actions prioritaires de la province Sud du 25 mars.

10. Voir schéma de mise en valeur des richesses minières (GNC, 2009, p. 54) : la SLN a un patrimoine de titres totalisant $1400 \mathrm{~km}^{2}$, soit environ 7,5\% de la superficie totale de la NouvelleCalédonie.

11. Le schéma de mise en valeur des richesses minières de la Nouvelle-Calédonie avait préconisé la mise en place d'un "Fonds Nickel ", " en remplacement du Fonds Conjoncturel du Secteur Minier actuel». Ce fonds devait remplir les fonctions suivantes : «(i) apporter une aide aux communes pour pallier les effets d'une éventuelle fermeture de centre minier ; (ii) soutenir les entreprises minières et leurs sous-traitants, notamment les rouleurs, dans les conjonctures de crise économique ; (iv) financer des programmes de réhabilitation des zones dégradées. » (GNC, 2009 , p. 180). On comprend donc que ce fonds devait avoir une nature essentiellement contracyclique et non au profit des générations futures.

12. À l'occasion de la séance publique du congrès de la NC du 18 mars 2009, M. Bretegnier avait ainsi déclaré : « Les 250 millions plafonnés, à l'époque, c'était assez logique de les plafonner parce que, effectivement, les collectivités, dont la Nouvelle-Calédonie et les provinces, avaient des difficultés budgétaires et, donc, il fallait en quelque sorte partager le peu qu'il y avait de recettes nickel. Là, d'un seul coup, on s'est trouvé avec une masse incroyable de recettes en provenance du nickel et c'est là qu'il ne fallait pas plafonner bien sûr, au contraire, il fallait déplafonner. (...) La marge de manœuvre on l'avait depuis 2004 jusqu'à 2009. C'est alors qu'on avait toutes les marges de manœuvre possibles et c'est alors qu'on pouvait faire ce fonds et c'est à ce moment-là qu'on pouvait travailler pour les générations futures. Il n'était pas besoin de gaspiller l'argent, de le jeter par les fenêtres.»

13. On pourrait cependant nuancer ces critiques en considérant que si, certes, la décision de 2004 était «imprévoyante», elle avait aussi pour objet de financer des besoins immédiats, liés à des politiques sociales dont le pays avait besoin. On peut aussi considérer que la dépense immédiate, dans la politique du logement, la politique de formation ou la politique familiale, facilitera à moyen terme l'accès au travail pour les populations en difficultés. Enfin, si au moment de la crise en 2009, certains ont pu regretter, que le fonds nickel n'ait pas pu disposer de davantage de fonds, du fait du plafond de 1,5 milliard décidé en 2004, la suite des événements a montré que la profession a pu passer la période de crise sans que l'on épuise pour autant les fonds disponibles.

14. Il faut noter qu'une redevance superficiaire est déjà instituée par le Code minier de NouvelleCalédonie de 2009 : «La redevance superficiaire due par le titulaire d'une concession minière est égale au produit de la surface réelle de la concession minière, arrondie à l'hectare supérieur, par un tarif à l'hectare. » (Article R. 131-3 du Code minier).

15. L'idée étant, qu'à l'issue du pacte fiscal, les sociétés métallurgiques « devraient » payer de l'IS 35 et donc contribueront ainsi à alimenter le fonds. Ces recettes permettraient de réduire les prélèvements sur la VA des autres secteurs.

16. Cf. Varii Auctores, Les déchets toxiques en quantité dispersée (DTQD) et l'eau, Bulletin de l'Union des Physiciens nº 808, Administration et Rédaction Editions, Paris, Novembre 1998. 


\section{RÉSUMÉS}

La Nouvelle-Calédonie possède entre 20 et $25 \%$ des réserves mondiales en nickel. Pour autant, bien que l'exploitation dure depuis plus de 130 ans, la rente minière profite peu aux populations et encore moins aux générations futures (alors que la ressources est par nature nonrenouvelable). Cet article analyse les raisons des blocages récurrents à la mise en place d'une épargne intergénérationnelle en Nouvelle-Calédonie, insiste sur la nécessité d'instaurer un tel dispositif basé sur la ressource minière et présente des solutions sur le modèle $d u$ «fonds pétrolier » norvégien.

New Caledonia owns from 20 to $25 \%$ of the world's reserves of nickel. However, although the mining exploitation has been running for more than 130 years, the mining rent is of little benefit to the people and even less to future generations (despite the fact that the resource is of a nonrenewable nature). This paper analyzes the reasons of the impediments to the implementation of an intergenerational saving scheme in New Caledonia and emphasizes the need for setting up such a mining resource-based mechanism, while presenting solutions along the lines of the Norwegian "petroleum fund".

\section{INDEX}

Keywords : New Caledonia, sustainable development, nickel, finance, fund for future generations, political cycles

Mots-clés : Nouvelle-Calédonie, développement durable, nickel, fiscalité, fonds pour les générations futures, cycles politiques

\section{AUTEURS}

\section{GAËL LAGADEC}

Gaël Lagadec est MCF en économie à l'Université de la Nouvelle-Calédonie. Ses thèmes de recherche sont l'économie publique et l'économie régionale. Lagade@univ-nc.nc

\section{OLIVIER SUDRIE}

Olivier Sudrie est Maître de conférences à l'Université de Versailles-Saint-Quentin-en-Yvelines. Ses thèmes de recherche concernent la politique économique, la modélisation macroéconomique et la prévision budgétaire. 